\title{
Goniodysgenesis-associated glaucoma in a Jindo dog
}

\author{
Manbok Jeong ${ }^{1 \%}$, Kristina Narfström ${ }^{2}$ \\ ${ }^{1}$ Woori Animal Medical Center, Seoul 08547, Korea \\ ${ }^{2}$ Department of Veterinary Medicine and Surgery, College of Veterinary Medicine, University of Missouri-Columbia, \\ Columbia, MO 65211, USA
}

\begin{abstract}
A 10-year-old intact female Jindo dog was presented with a 1-week history of conjunctival redness and ocular discharge in the left eye. There was an absence of menace response, dazzle reflex, and direct pupillary light reflex. Slit-lamp biomicroscopy revealed corneal edema, ciliary flush, and aqueous flare. Intraocular pressure was $68 \mathrm{mmHg}$. Based on the information available, a diagnosis of glaucoma and uveitis was made. Subsequent histopathologic examination showed the glaucoma was produced by the effects of goniodysgenesis, posterior synechia, and pigment dispersion in the trabecular meshwork. This is the first report of primary glaucoma caused by goniodysgenesis in Jindo dogs.
\end{abstract}

Keywords: dog, glaucoma, goniodysgenesis, Jindo

*Corresponding author

Manbok Jeong

Woori Animal Medical Center, 1386

Nambusunhwan-ro, Geumcheon-gu, Seoul 08547, Korea

Tel: $+82-2-853-7582$

Fax: +82-2-858-7583

E-mail: 9757044@hanmail.net

ORCID:

Manbok Jeong

https://orcid.org/0000-0002-0155-1109

Conflict of Interest

The authors declare no conflicts of interest.

Received: February 26, 2019

Revised : May 8, 2019

Accepted: May 27, 2019
Canine glaucoma induces progressive loss of vision resulting from death of retinal ganglion cells and their axons due to abnormally increased intraocular pressure (IOP) [1]. It can be classified based on clinical cause (congenital, primary, or secondary) and by evaluation of the iridocorneal angle (ICA) using gonioscopy (open, narrow, or closed).

Primary glaucoma can be subdivided into primary open-angle glaucoma (POAG) and angle-closure glaucoma (PACG) [1]. Goniodysgenesis (GD) and pectinate ligament dysplasia (PLD) have been included within the range of abnormalities of the pectinate ligament. Therefore, both PACG and GD have been used together to describe the morphologic abnormalities of the ICA. Many canine breeds have been reported to be affected by PACG [2].

The prevalence of primary glaucoma is firmly associated with geographic differences and other factors including higher IOP, age, genetics, vascular factors, immunologic factors, and environmental factors [1]. Several researchers have reported on glaucoma populations in dogs based on the prevalence of the possible geographic differences [3,4]. There is only one previous report regarding the ophthalmic disease in Jindo dogs (registered a Korean Natural Monument number 53) but without a description of primary glaucoma [5]. Therefore, the purpose of the present study was to report GD-associated primary glaucoma identified histopathologically in a Jindo dog.

A 10-year-old intact female Jindo dog was presented with a 1-week history of conjunctival redness and ocular discharge in the left eye (OS). On presentation, buphthalmos, mucoid discharge, and mild blepharospasm were obvious in OS. Menace response, dazzle reflex, and direct pupillary light reflexes (PLRs) were absent in OS. Indirect PLRs from OS to the right eye (OD) were also absent. On slit-lamp biomicroscopy (HS-7000; Huvitz Co., Ltd., Korea), marked episcleral vascular injection, diffuse corneal edema, and circum-corneal ciliary flush were noted in OS, along with moderate $(3+)$ flare and dyscoria accompanied by rubeosis iridis and iris bombé (Fig. 1). Fluorescein staining showed no corneal defects. IOPs measured by rebound tonometry (TonoVet ${ }^{\mathbb{R}}$; Icare/Tiolat, Finland) were $20 \mathrm{mmHg}$ OD and $68 \mathrm{mmHg}$ OS. Indirect ophthalmoscopy (Vantage Plus ${ }^{\circledR}$; Keeler Instruments Inc., USA) and gonioscopy (Koeppe large diagnostic lens; Ocular Instruments Inc., USA) of OS were attempted, but it was impossible to observe the fundus and ICA due to corneal, anterior 


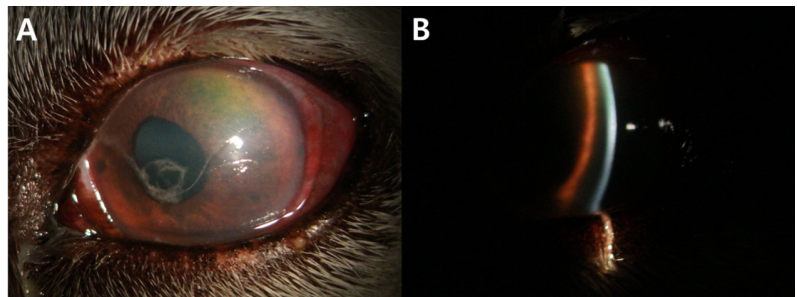

Fig. 1. Ocular appearance of the left eye based on slit-lamp biomicroscopy at first presentation. (A) Image by direct diffuse illumination. Note the diffuse corneal edema, circum-corneal ciliary flush, dyscoria, and ectropion uvea. (B) Image by direct focal illumination. Note the aqueous flare indicating uveitis.

chamber problems (Fig. 1). Gonioscopy of OD showed a partially narrowed ICA. The ophthalmic examination results allowed us to diagnose the dog with both glaucoma and uveitis OS. There were no abnormalities OD except a partially narrowed ICA at initial presentation.

Topical medication of OS included i) $2 \%$ dorzolamide $\mathrm{HCl}$ (Trusopt $^{\circledR}$; Laboratoires Merck Sharp \& Dohme-Chibret, France; q8h), ii) $0.5 \%$ levofloxacine $\left(\right.$ Cravit $^{\circledR}$; Santen Pharmaceutical Co., Ltd., Osaka, Japan; q8h), and iii) 1\% prednisolone acetate (Pred Forte ${ }^{\circledR}$; Allergan Pharmaceuticals, Ireland; q6h). Prophylactic treatment of OD consisted of $0.5 \%$ Betaxolol (Betoptic $^{\circledR}$; Alcon-couvreur N.V., Puurs, Belgium; q12h).

The dog was re-examined 1 week after initial presentation at our clinic. The owner reported that there was no significant improvement compared to the first presentation and that there were difficulties in instillation of the topical medication at home. Enucleation was recommended based on the owner's poor compliance and poor prognosis. Routine blood work prior to surgery was unremarkable. Subconjunctival enucleation was performed 2 weeks after the first presentation. The globe was immersed in formalin and sent to the Comparative Ocular Pathology Laboratory of Wisconsin at the University of Wisconsin, USA for histopathologic analysis. Prophylactic treatment of OD was continued with $0.5 \%$ Betaxolol (q12h). The fellow eye developed clinical glaucoma 2 years and 5 months after the OS developed glaucoma.

Histologically, the OS anterior and posterior chambers and the vitreous contained a lightly eosinophilic proteinaceous material. The iris profile was thin and bowed forward and a dense fibrovascular membrane lined the anterior lens capsule, which was consistent with iris bombé. There were scattered lymphocytes and plasma cells in the iris stroma. The ICA was distorted by an iris-like tissue extending from the base of the iris, crossing the ICA and attaching to the arborized end of Descemet's membrane, observations typical of GD. The ciliary cleft and trabecular meshwork contained numerous pigmented cells and free melanin granules (pigment dispersion) (Fig. 2A). The limbal sclera was thin. There was retinal atrophy with tapetal sparing and the optic nerve head was severely cupped, gliotic, and atrophied (Fig. 2B). The present case was histopathologically confirmed as glau-

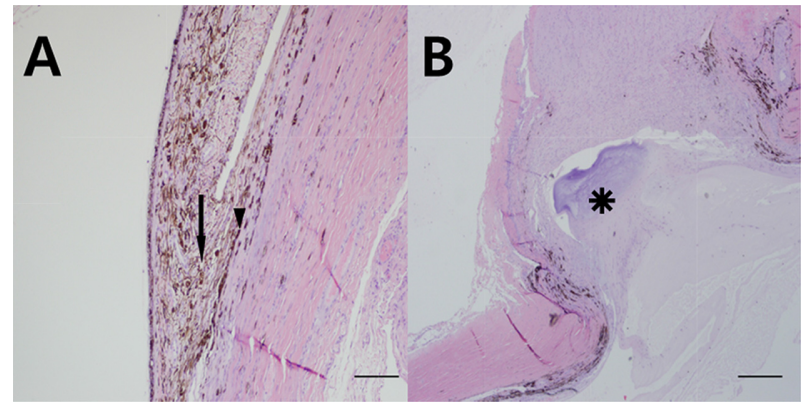

Fig. 2. Histopathological evaluation with $H \& E$ staining. (A) The iridocorneal angle is distorted by an iris-like tissue extending from the base of the iris, crossing the iridocorneal angle (arrow), and attaching to the arborized end of Descemet's membrane (arrowhead). The ciliary cleft and trabecular meshwork contain numerous pigmented cells and free melanin granules (pigment dispersion). (B) The optic nerve head is severely cupped, gliotic, and atrophied (asterisk). Scale bars $=20 \mu \mathrm{m}$ (A) and $50 \mu \mathrm{m}(\mathrm{B})$.

coma based on the associated effects of GD, posterior synechia, and pigment dispersion in the trabecular meshwork.

This case study is, to the best of the authors' knowledge, the first report of primary glaucoma caused by GD in the Jindo dog breed. Primary glaucoma is hereditary in many canine breeds and is defined as an elevation of IOP not connected any other pre-existing ocular disease [1]. Frequently, PACG is also related to congenital abnormalities of the pectinate ligament known as PLD or GD. In secondary glaucoma, the increase in IOP is associated with antecedent or concurrent ocular disease, such as uveitis and lens luxation/ subluxation that can physically block aqueous outflow [1]. The clinical presentation described in this report allowed us to diagnose the ocular disease as secondary glaucoma because gonioscopic evaluation of the pectinate ligament was precluded by moderate corneal edema and inflammatory cells at the level of the anterior chamber. However, the suspicion of primary glaucoma arose based on the gonioscopically observed narrowed ICA of the unaffected eye (OD).

The histopathologic findings described above are similar to those in previous reports related to histopathological GD; for example, uveal inflammatory infiltrates and pigment dispersion, which are present in the majority of dogs with primary glaucoma [2,6]. GD has been described in many canine breeds; however, the Jindo dog has not been included in the GD group until this case [1]. Based on the previous investigation, in the present report of a Jindo dog, the presence of GD together with inflammatory changes such as posterior synechia and pigment dispersion might have contributed to the development of glaucoma OS [7].

With GD, once glaucoma develops in one eye, the fellow eye is at risk of the development of glaucoma within weeks to years, sometimes within a matter of days [2]. For this reason, many studies have reported on the necessity of prophylactic antiglaucoma medications for the fellow eye [8,9]. In 
this case report, the fellow eye OD developed glaucoma 2 years and 5 months after development of glaucoma OS despite the use of prophylactic antiglaucoma medication $(0.5 \%$ Betaxolol, q12h). However, the preventive medication was likely to have delayed the onset of glaucoma OD on the basis of the 2-year and 5-month intervals from initiation of medical therapy. In the present report, both the diagnosis of GD by histopathologic examination and the development of glaucoma in the contralateral eye should be enough to validate that the canine breed can exhibit primary glaucoma.

We suggest here the possibility of the presence of primary glaucoma associated with GD, posterior synechia, and pigment dispersion in the trabecular meshwork in the Korean Jindo dog population (Korean Natural Monument number 53). Therefore, the clinical prevalence of GD-associated glaucoma needs to be investigated in this Korean canine breed.

\section{References}

1. Pizzirani S. Definition, classification, and pathophysiology of canine glaucoma. Vet Clin North Am Small Anim Pract 2015;45:1127-1157.

2. Reilly CM, Morris R, Dubielzig RR. Canine goniodysgenesisrelated glaucoma: a morphologic review of 100 cases looking at inflammation and pigment dispersion. Vet Ophthalmol 2005; 8:253-258.
3. Gelatt KN, MacKay EO. Prevalence of the breed-related glaucomas in pure-bred dogs in North America. Vet Ophthalmol 2004;7:97-111.

4. Strom AR, Hässig M, Iburg TM, Spiess BM. Epidemiology of canine glaucoma presented to University of Zurich from 1995 to 2009. Part 1: congenital and primary glaucoma (4 and 123 cases). Vet Ophthalmol 2011;14:121-126.

5. Seo KM, Jang KH, Cheong JT, Yeon SC, Lee GW, Nam TC. A survey of ophthalmic diseases in Korean Jindo dogs. J Vet Clin 2001;18:215-225.

6. Pumphrey SA, Pizzirani S, Pirie CG, Needle DB. Glaucoma associated with uveal cysts and goniodysgenesis in American Bulldogs: a case series. Vet Ophthalmol 2013;16:377-385.

7. Beamer G, Reilly CM, Pizzirani S. Microscopic lesions in canine eyes with primary glaucoma. Vet Clin North Am Small Anim Pract 2015;45:1213-1233.

8. Dees DD, Fritz KJ, Maclaren NE, Esson DW, Sheehan Gaerig AM, Atkins RM, Knollinger AM. Efficacy of prophylactic antiglaucoma and anti-inflammatory medications in canine primary angle-closure glaucoma: a multicenter retrospective study (2004-2012). Vet Ophthalmol 2014; 17:195-200.

9. Miller PE, Schmidt GM, Vainisi SJ, Swanson JF, Herrmann MK. The efficacy of topical prophylactic antiglaucoma therapy in primary closed angle glaucoma in dogs: a multicenter clinical trial. J Am Anim Hosp Assoc 2000;36:431-438. 